\title{
Percepção docente sobre a Aprendizagem Baseada em Problemas no ensino remoto durante a pandemia COVID-19
}

\author{
Faculties' perception about Problem-Based Learning in remote education during pandemic
} COVID-19

\author{
Percepción de los docentes sobre el Aprendizaje Basado en Problemas en la educación remota \\ durante la pandemia COVID-19
}

\section{Resumo}

O grande desafio das escolas médicas durante a pandemia da COVID-19 foi fazer a transição, em curto período, do ensino presencial da Aprendizagem Baseada em Problemas (ABP) para o ensino remoto de emergência (ERE). Este trabalho teve como objetivo relatar os resultados da implementação da ABP no ERE no curso de Medicina de um Centro Universitário em Fortaleza. Trata-se de um estudo de corte transversal, descritivo e quantitativo. Um formulário eletrônico no formato Google Docs, enviado a todos os docentes após conclusão das atividades de tutoria do primeiro semestre letivo de 2020, avaliou sua percepção quanto à eficácia da ABP no ERE, às dificuldades de adaptação à modalidade de ERE, à sobrecarga cognitiva das sessões tutoriais e aos fatores interferentes na interação tutor-aluno online. Os tutores assinaram um termo de consentimento e o anonimato foi garantido. Responderam ao formulário 89 tutores, $60,7 \%$ do sexo feminino, idade média de 40,9 anos e mediana do tempo de experiência como tutor de dois anos. Quanto à eficácia do ABP no ERE, 49,4\% dos tutores julgaram ser menor em relação ao ensino presencial; 70,8\% não tinha nenhuma/mínima experiência com tecnologias educacionais antes da pandemia; $46,1 \%$ tiveram dificuldades de adaptação na modalidade de ERE; 69,6\% evidenciaram a sobrecarga cognitiva dos discentes e $74,1 \%$ concordaram com a necessidade de diminuição da carga horária da tutoria. Os principais fatores que interferiram na interação tutor-aluno foram interrupção da rede de internet, câmera desligada e comunicação não verbal deficiente. Portanto, a utilização de ABP no ERE deve ser realizada de forma modificada, com diminuição das horas de atividades síncronas, com melhor capacitação dos tutores e alunos quanto às TICs, melhor conhecimento das avaliações interativas online e das medidas profiláticas da fadiga zoom.

Palavras-chave: Ensino; Educação à distância; Aprendizagem baseada em problemas; Pandemia.

\section{Abstract}

The great challenge for medical schools during the COVID-19 pandemic was to make the transition, in a short period, from face-to-face teaching of Problem Based Learning (PBL) to remote emergency teaching (ERT). This paper had 
the objective to report the results of the implementation of the PBL in the ERT in the medical faculty of a University Center in Fortaleza. This is a cross-sectional, descriptive and quantitative study. An electronic form in Google Docs format, sent to all teachers after completing the tutoring activities in the first academic semester of 2020, assessed their perception of the effectiveness of PBL in the ERT, the difficulties of adapting to this modality, the cognitive overload of tutorial sessions and the interfering factors in the online tutor-student interaction. The docents signed a consent form and anonymity was guaranteed. 89 tutors responded to the form, $60.7 \%$ were female, with an average age of $40.9 \mathrm{ys}$ and the median time of experience as a tutor was two-years. Regarding the effectiveness of PBL in the ERT, $49.4 \%$ of the tutors considered it was lower compared to classroom teaching; $70.8 \%$ had no/minimal experience with educational technologies before the pandemic; $46.1 \%$ had difficulties in adapting to the ERT modality; $69.6 \%$ evidenced the students' cognitive overload and $74.1 \%$ agreed with the need to reduce the tutoring hours. The main factors that interfered in the tutor-student interaction were interruption of the internet network, camera turned off and deficient non-verbal communication. Therefore, the use of PBL in the ERT should be carried out in a modified way, with reduced hours of synchronous activities, with better training of tutors and students in TICs, better knowledge of interactive online assessments and prophylactic measures of zoom fatigue.

Keywords: Teaching; Education distance; Problem-based learning; Pandemics.

\section{Resumen}

El mayor desafío para las escuelas de medicina durante la pandemia de COVID-19 fue hacer la transición, en un corto período, de la enseñanza presencial del Aprendizaje basado en problemas (ABP) a la Enseñanza de emergencia remota (EER). Este trabajo tuvo como objetivo reportar los resultados de la implementación del PBL en el EER en el curso de Medicina de un Centro Universitario en Fortaleza. Se trata de un estudio transversal, descriptivo y cuantitativo. Un formulario electrónico en formato Google Docs, enviado a todos los docentes tras completar las actividades de tutoría en el primer semestre académico de 2020, evaluó su percepción de la efectividad del ABP en los ERE, las dificultades de adaptación a la modalidad EER, la sobrecarga cognitiva de las tutorías y los factores que interfieren en la interacción en línea tutor-alumno. Los tutores firmaron un formulario de consentimiento y se garantizó el anonimato. 89 tutores respondieron al formulario, el 60,7\% eran mujeres, con una edad media de 40,9 años y una mediana de dos años de experiencia como tutor. En cuanto a la efectividad del ABP en el EER, el 49,4\% de los tutores la considera menor en relación a la docencia en el aula; el 70,8\% tenía poca o ninguna experiencia con tecnologías educativas antes de la pandemia; el 46,1\% tuvo dificultades para adaptarse a la modalidad EER; el 69,6\% evidenció sobrecarga cognitiva de los estudiantes y el 74,1\% estuvo de acuerdo con la necesidad de reducir las horas de tutoría. Los principales factores que interfirieron en la interacción tutor-alumno fueron la interrupción de la red de internet, la cámara desconectada y la comunicación no verbal deficiente. Por tanto, el uso del ABP en los ERE debe llevarse a cabo de forma modificada, con una reducción de las horas de actividades sincrónicas, con una mejor formación de los tutores y alumnos en las TICs, un mejor conocimiento de las evaluaciones interactivas online y medidas profilácticas de fatiga del zoom.

Palabras clave: Ensenãnza; Educación a distancia; Aprendizaje basado en problemas; Pandemias.

\section{Introdução}

O grande desafio das escolas médicas durante a pandemia da COVID-19 foi fazer a transição, em curto período, do ensino presencial para o ensino remoto de emergência (ERE), a fim de reduzir os danos educacionais. A demanda de soluções rápidas e, por vezes, improvisadas, realizadas em circunstâncias desfavoráveis, determinou inquietações e reflexões (Goldhome, et al., 2020; ROSE, 2020; Tempski, et al., 2020; Gibbs, 2020; Tolsgaard, et al., 2020; Hodges, et al., 2020). Tal fato traduz-se na mudança de concepção do novo modo de ensinar. A implementação da Aprendizagem Baseada em Problemas (ABP) também representou desafios, devido à falta de prática dos tutores no formato remoto. Essa inexperiência pode interferir no processo pedagógico no que se refere às avaliações formativa e somativa; à administração do tempo; à motivação e ao envolvimento dos alunos e socialização online, entre outros aspectos (Bizarria, et al., 2013).

No entanto, a ABP pode ser aplicada de forma eficaz na educação remota, seguindo os passos da metodologia, que incluem a apresentação do problema, a aprendizagem autodirecionada, a investigação, a discussão, a tomada de decisões e o trabalho cooperativo (Mezzari, 2011). É importante ressaltar que a preparação adequada do AVA necessitaria de meses de planejamento e design cuidadoso, usando um modelo sistemático de design e desenvolvimento (Taylor, et al., 2020).

Desta forma, a análise da experiência didático-pedagógica, utilizando a ABP em um cenário de ensino virtual, até então não vivenciado pelo corpo docente e com tempo de preparação mínimo, faz-se de suma importância para compreender 
melhor as fortalezas e dificuldades percebidas. O acompanhamento da nova dinâmica de ensino permite a construção de indicadores que auxiliem nas tomadas de decisões e no planejamento estratégico futuro.

O Problem Based-Learning (PBL) ou Aprendizagem Baseada em Problemas (ABP) tem uma origem conceitual e filosófica inspirada em Jerome Bruner, Jonh Dewey e Jean Piaget (Borges, et al., 2014; Torre, van der Vleuten, Dolmers, 2016). Há uma grande variedade de métodos de implantação (Al-Dous \& Samaka, 2015). No curso de Medicina em questão, o ABP tem como inovação na metodologia a adição de dois passos, aos sete passos do método tradicional (1. esclarecer termos e expressões desconhecidos no texto do problema; 2. listar os problemas; 3. analisar e discutir os problemas, com explicações para os mesmos baseado no conhecimento prévio (chuva de ideias); 4. resumir as explicações, sistematizando a análise e hipóteses do problema; 5.formular os objetivos de aprendizagem para aprofundamento e complementação das explicações; 6 . buscar informações e realizar estudo individual respeitando os objetivos de aprendizagem; 7. integrar as informações e rediscutir o caso com base nos avanços do conhecimento obtido pelo grupo). O passo oito (8) consiste na apresentação por cada aluno de um artigo científico relacionado com o conteúdo do caso problema, identificado na busca individual da literatura, e o passo nove (9) representa uma avaliação cognitiva por meio de um teste escrito com a utilização de casos clínicos, simulando problemas a serem analisados pelo discente, denominado de "caso integrador". Este provê valiosa informação sobre a efetividade dos objetivos de aprendizagem estabelecidos nos problemas (Kelley, et al., 2019; Ribeiro \& Escrivão, 2011).

Portanto, o presente estudo teve como objetivo relatar os resultados da implementação da ABP no ERE no curso de Medicina de um Centro Universitário em Fortaleza, durante a pandemia da COVID-19.

\section{Metodologia}

Trata-se de um estudo de corte transversal, descritivo e quantitativo, realizado em um curso de Medicina de Fortaleza. Durante a pandemia, a ferramenta de apoio escolhida pelo curso de Medicina do Centro Universitário Christus para o ensino virtual foi o Moodle (Modular Object-Oriented Dynamic Learning Enviroment), por ser um software livre e já utilizado. Através desta plataforma, todos os discentes e docentes acessaram o Google Meet institucional, para a realização das sessões tutoriais. Além disso, foi montado um serviço de apoio técnico contínuo, com capacitações para todo o corpo acadêmico.

Em junho de 2020, ao término do primeiro semestre do ano letivo, foi enviado um formulário eletrônico no formato Google Docs, por whatsapp, a todos os tutores da ABP (do primeiro ao oitavo semestre), como um convite para colaborar no entendimento da experiência inicial com o ERE do método tutorial, em virtude da pandemia da COVID-19. O anonimato dos tutores foi preservado, não constando seus nomes nos formulários. O Termo de Consentimento Livre e Esclarecido foi assinado eletronicamente. O projeto de pesquisa foi submetido ao Comitê de Ética do Centro Universitário Christus e aprovado sob o número de Parecer: 4.079.785.

As perguntas do questionário eletrônico foram elaboradas pelos autores da pesquisa e pretendiam conhecer a percepção dos tutores quanto à eficácia da ABP no ERE, às dificuldades de adaptação à modalidade de ERE, à sobrecarga cognitiva das sessões tutoriais e aos fatores interferentes na interação tutor-aluno online. O formulário eletrônico continha perguntas fechadas e abertas.

Por meio das respostas obtidas, os dados foram transferidos para uma planilha de Excel 13.0, e, posteriormente, para o SPSS 23.0, para análise dos resultados. As variáveis contínuas foram expressão em média e desvio padrão ou medianas e percentis 25 e 75 e as variáveis categóricas foram expressas em percentuais. 


\section{Resultados}

Responderam ao formulário eletrônico 89 tutores do primeiro ao oitavo semestre (representando 99\% dos docentes), com média de idade de 40,9 $\pm 10,6$ anos (variação $=28-69$; mediana $=37$ ), sendo $60,7 \%$ do sexo feminino $(n=54)$. A mediana do tempo de experiência como tutor foi de dois anos (variação de 0,5-13 anos). No grupo pesquisado, 92,1\% eram médicos $(n=82), 4,5 \%$ farmacêuticos $(n=4)$ e 3,4\% biólogos $(n=3)$. A distribuição dos tutores por semestre encontra-se no Gráfico 1. Observa-se que o percentual de tutores até o sexto semestre foi mais elevado do que o sétimo e oitavo semestre, porque até o sexto semestre o curso tem o dobro de alunos e tutores, em relação a sétimo e oitavo semestres, em função de ampliação de vagas do curso, que ainda não alcançou os últimos semestres.

Gráfico 1. Distribuição dos tutores participantes da pesquisa, segundo o semestre letivo.

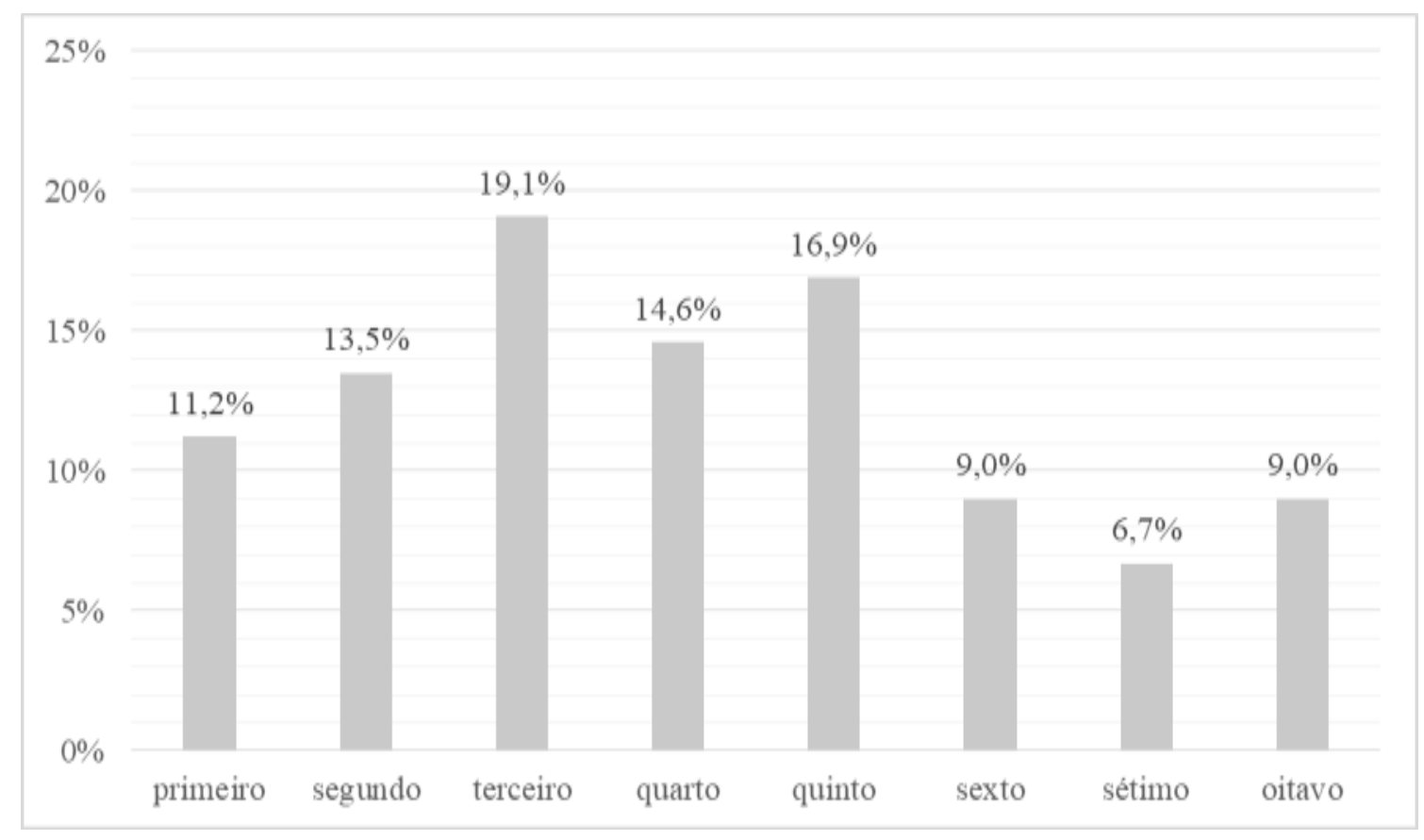

Fonte: Autores.

Sobre a eficácia da ABP por meio do ensino remoto comparada ao ensino presencial, as respostas obtidas sinalizaram que $49,4 \%$ dos tutores discordaram ou discordaram totalmente, e 58,4\% dos tutores conseguiram seguir os passos da tutoria totalmente e $41,6 \%$ o fizeram parcialmente.

A experiência prévia com as tecnologias educacionais à distância foi informada como nenhuma em $52,8 \%$ dos casos, mínima em 18,0\%, regular em 19,1\%, moderada em 6,7\% e ótima em 3,4\%. Dificuldades iniciais na adaptação à modalidade de ensino remoto foram referidas por $46,1 \%$ dos tutores. $87,7 \%$ concordaram ou concordaram totalmente quanto à necessidade de câmeras ligadas na sessão tutorial. Sobrecarga ou fadiga cognitiva dos discentes no ensino remoto foi registrada por 69,6 \% dos tutores em suas respostas. É provável que essa percepção justifique que, quando questionados se o tempo de tutoria na tutoria remota deve ser menor que na presencial, $74,1 \%$ concordaram ou concordaram totalmente.

Em relação à avaliação formativa dos alunos, somente 33,7\% concordaram ou concordaram totalmente que ela é eficaz no ensino remoto. Além disso, 47,2\% dos tutores concordaram ou concordaram totalmente que a avaliação do conhecimento por meio do caso integrador é um instrumento de avaliação que tem pouca interferência da modalidade a ser aplicada (remota ou presencial). Um sumário das respostas dos tutores ao formulário eletrônico encontra-se no Gráfico 2. 
Research, Society and Development, v. 10, n. 5, e53510515280, 2021

(CC BY 4.0) | ISSN 2525-3409 | DOI: http://dx.doi.org/10.33448/rsd-v10i5.15280

Gráfico 2. Avaliação das respostas dos tutores ao formulário eletrônico, segundo escala de Likert.

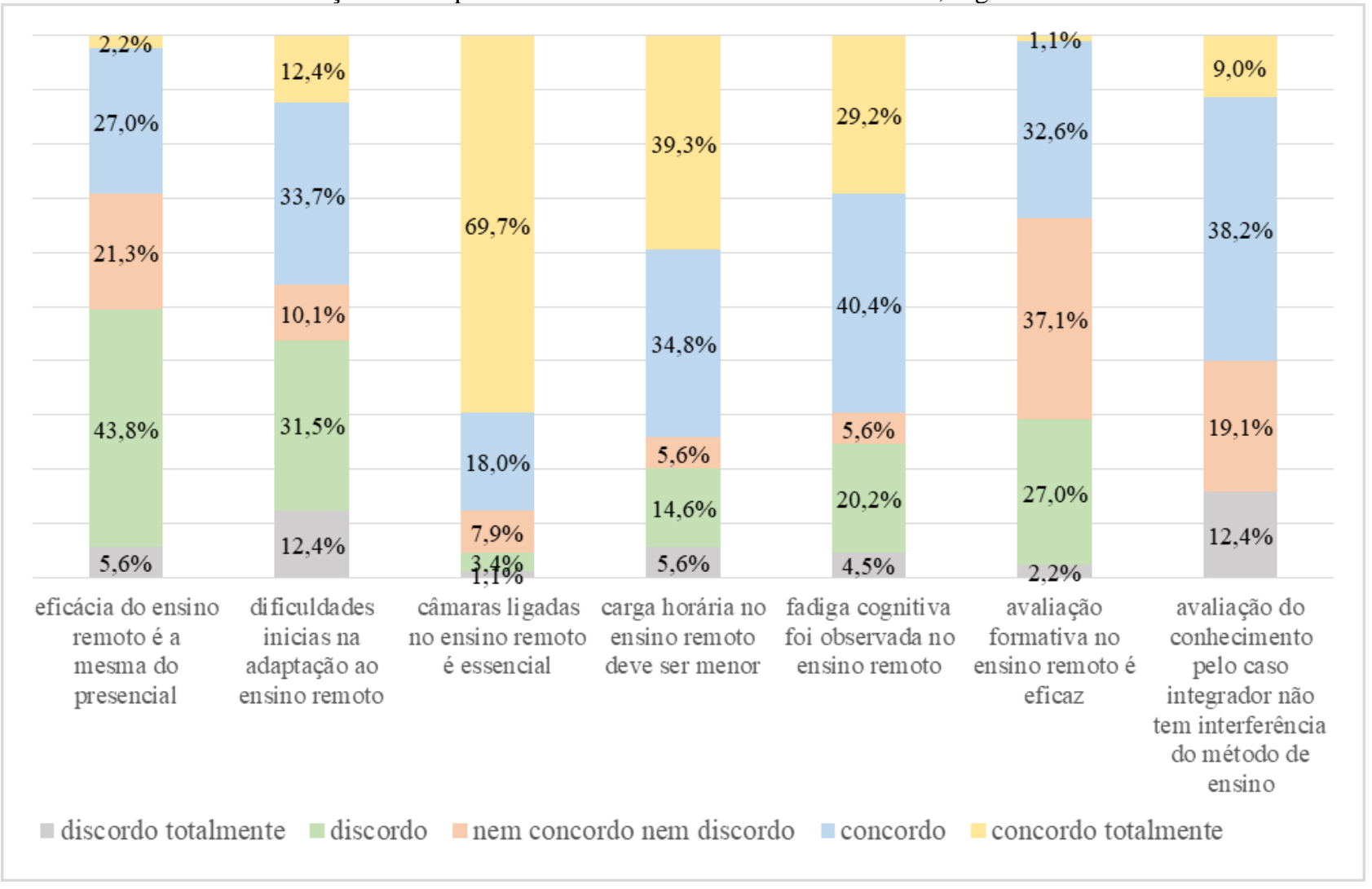

Fonte: Autores.

Os principais fatores que interferiram na interação tutor-aluno no ensino remoto, segundo os tutores, estão apresentados no Gráfico 3.

Gráfico 3. Fatores que interferiram na interação tutor-aluno no ensino remoto, segundo os tutores.

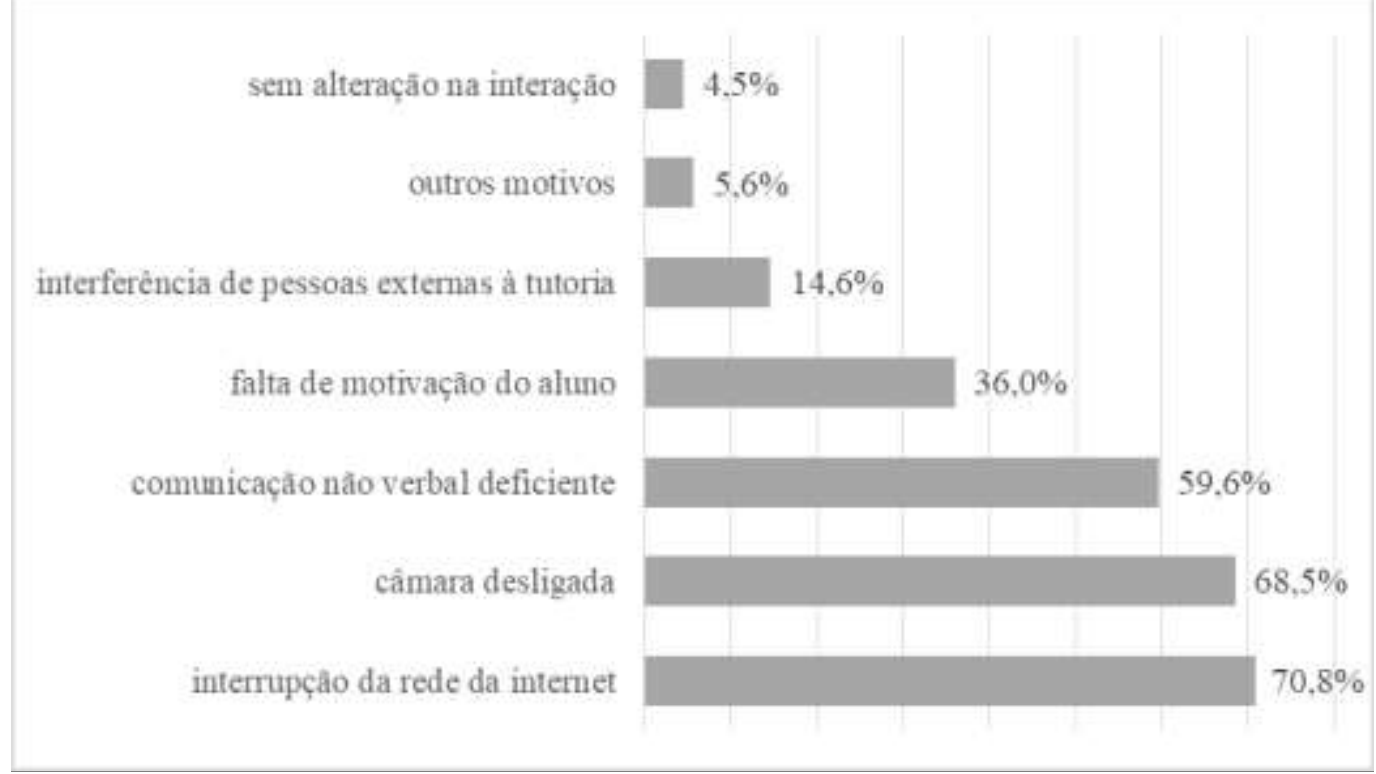

Fonte: Autores.

Dentre as dificuldades referidas para os passos da ABP remota, destacam-se a apresentação monótona, sem interação dos demais alunos, e a dificuldade de manter a atenção do grupo. Alguns tutores referiram que usaram seminários relacionados 
ao caso e artigos com quizz para contornar as dificuldades. Em relação ao passo 4 (organização e exposição das hipóteses explicativas), alguns tutores relatam que a menor motivação e estudo dos alunos pode ter contribuído para o problema.

\section{Discussão}

Houve baixa percepção de eficácia da ABP realizada no ERE pelos docentes. Observou-se que quase a metade dos tutores não conseguiu executar todos os passos como no formato presencial, com dificuldade de adaptação ao novo formato pelos tutores. Um desafio importante para futuros programas de desenvolvimento docente é a pouca experiência com o uso de plataformas e ferramentas online pela maioria dos tutores. Esses achados oriundos de uma ação emergencial, sem planejamento prévio, corroboram os relatos de David Taylor (Taylor, et al., 2020), o qual afirma que qualquer processo de mudança em si precisa, para seu êxito de um planejamento prévio cuidadoso, de professores ativos e motivados em tecnologia, plataformas de fácil utilização, recursos de internet disponíveis e treinamento discente e de pessoal técnico para apoiar e gerenciar o processo, entre outras necessidades.

Além disso, para que os tutores possam exercer suas atividades com bom desempenho no AVA, neste período ora vivenciado, seriam necessários cursos de capacitação que proporcionassem conhecimento em Tecnologia da Informação e Comunicação (TIC), habilidades sociais online e ensino criativo, desenvolvendo, assim, um estilo de ensino pessoal no ambiente virtual (Hampel \& Stickler, 2005). A falta da execução dessas recomendações nos procedimentos pedagógicos durante a pandemia, como treinamento prévio dos alunos e disponibilidade de recursos de internet a todos, justifica, também, alguns fatores assinalados pelos tutores como fatores negativos na interação social no ERE, como interrupção da rede da internet e falta de motivação dos alunos (Silva, Sousa, Menezes, 2020).

Em relação às câmeras ligadas durante a sessão tutorial online, a maioria dos tutores concordam com a necessidade de deixá-las ligadas durante a tutoria, sob risco de prejuízo na interação tutor-aluno online. Esses achados vão de encontro aos relatos da literatura, ou seja, as câmeras ligadas favorecem a interação social, componente essencial para aprendizagem na ABP (Borges, et al., 2014). O uso da câmera é uma forma de monitorar e envolver todos em uma participação efetiva na discussão em grupo, evitando, também, a perda de foco decorrente das multitarefas (Office Academic Affairs, 2020; Sacasas, 2020).

Ademais, as câmeras ligadas estão implicadas positivamente no favorecimento da comunicação não verbal no AVA. Esta pista corporal por meio dos rostos tem um papel essencial uma vez que grande parte da comunicação humana ocorre mediante comunicação não verbal (linguagem corporal) e esta deve sempre estar sincronizada com a comunicação verbal para seu pleno funcionamento (Leonard, 2020). Enfim, escutar é, portanto, compartilhar o ver e o ouvir, é "aceitar o fato de se deixar impregnar pelo conjunto das percepções externas e internas" (Nobre \& Guerreiro, 2020).

Entretanto, as câmeras ligadas suprem apenas parcialmente a falta da comunicação não verbal, uma vez que o rosto do aluno fica visível para o tutor, mas, geralmente, a uma distância inferior à ideal, dificultando a percepção das dicas sutis em que confiamos para avaliar se alguém está interessado ou desengajado (Sacasas, 2020). Consequentemente, a falta da pista corporal plena (comunicação não verbal) no AVA torna-se um fator limitante para a interação online, conforme acordado pelos tutores desse estudo. Além disso, as videoconferências nos obrigam a ter um estilo de comunicação verbal não natural uma vez que, funciona com um fluxo único de áudio feito por apenas uma pessoa, para evitar a realimentação positiva do som, tornando assim, o ensino online menos colaborativo e mais lento (Office of Academic Affairs, 2020).

Vale ressaltar que a concentração por muito tempo nas imagens pixelizadas das telas pode levar a uma sobrecarga ocular e à Síndrome da Visão Computacional, resultante de problemas oculares e visuais relacionados ao uso do computador por períodos longos e ininterruptos de tempo (Innerdrive, 2019; Dodge, 2020). Pode cursar com fadiga, dor cervical, visão turva, ressecamento ocular, diplopia e dificuldade em focar novamente os olhos, afetando cerca de 9 entre 10 pessoas que 
permanecem três horas ou mais no computador, tempo que coincide com a duração da sessão tutorial. Nesse cenário, nosso cérebro precisa também fazer um esforço extra para compensar todos os aspectos de comunicação que faltam, levando a uma fadiga. Dessa forma, passou-se a recomendar o uso das câmeras, porém com desligamento em média de 20 segundos a cada 20 minutos (Sacasas, 2020).

A sobrecarga cognitiva ('fadiga zoom") foi observada pela maioria dos tutores. Esse achado vai de encontro aos registros da literatura que relatam este efeito adverso do AVA e os fatores implicados, entre outros: a deficiência da comunicação não verbal, exigindo uma atenção maior para ver as pessoas através de uma tela plana, vendo pequenos rostos; a dissonância (um sentimento e seu oposto), ou seja, a ilusão da presença, mas que, na realidade, há uma distância; a autoconsciência, ou seja, o vídeo sempre ligado a mostrar a aparência; a pressão social, que representa a sensação de que a pessoa precisa se apresentar; a dor, uma vez que as aulas remotas lembram vidas normais interrompidas durante a pandemia da COVID-19, e a perda da socialização, uma vez que todas as atividades sociais e de trabalho se dão em uma tela no "escritório (Petriglieri, 2020).

Diante do conhecimento que a existência adequada dos vários níveis de comunicação verbal e não verbal é um fator importante na retenção da aprendizagem e que a tentativa de mantê-la ao longo do ERE leva à fadiga e ao comprometimento do processo pedagógico, a maioria dos tutores $(74,1 \%)$ concordaram que a tutoria em ambiente virtual deveria ter uma duração menor do que a praticada no formato presencial. A tutoria presencial no nosso curso de medicina tinha a duração de 3,5 horas, e tendo como base o tempo da sessão tutorial descrita na literatura ( 2 horas), decidiu-se pela redução de uma hora da sessão tutorial cuja duração passou a ser 2,5 horas no formato online (Moust, Berkel, Schmidt, 2005; Pazin Filho \& Scarpelini, 2007)

Em relação à avaliação formativa dos alunos, somente $33,7 \%$ concordaram ou concordaram totalmente com o fato de ela ser eficaz. Tal percentual provavelmente decorre da falta de capacitação dos tutores para a utilização da interatividade da TIC e da deficiência de acompanhamento dos alunos nas atividades síncronas, como realizadas durante a pandemia. Essa deficiência, provavelmente, é agravada igualmente pelo desligamento das câmeras e pelo fato de a interação virtual ser feita na realidade, como um "monólogo", uma vez que, usualmente, quando uma pessoa fala, é solicitado às demais o desligamento dos microfones, evitando, assim, a microfonia. Isso contribui para que as conversas de vídeos sejam menos participativas. (Office Academic Affairs, 2020).

Prevendo alguma dificuldade com a manutenção da prática de feedback aos alunos, optou-se por valorizar o caso integrador, uma vez que, por ser uma avaliação de conhecimentos, tem uma menor interferência do modo remoto de ensinar. Assim, mantivemos este passo no nosso guia remoto, com um peso maior na avaliação da sessão tutorial. A Universidade de Sherbrooke, em reunião por webconferência, relatou ter preferido, neste início do ERE, não realizar a avaliação somativa e formativa na ABP.

Há lacunas importantes no desenvolvimento docente diante do cenário que se impôs. As dificuldades e estratégias desse período inicial permite planejar melhor as próximas etapas da modalidade remota, percebendo as lições aprendidas que devem permanecer, bem como os ajustes necessários. Aspecto central da experiência apresentada diz respeito às competências necessárias aos tutores para essa adaptação e para a definição do formato para o ensino médico pós-pandemia.

São limitações do estudo a realização da pesquisa somente em um único momento do semestre letivo, a não inclusão da opinião dos discentes, bem como a impossibilidade de realização de grupos focais presenciais onde as dificuldades e fortalezas do método on-line pudessem ter sido discutidas mais amplamente.

\section{Conclusão}

As considerações apontadas nesta pesquisa sugerem que a utilização de ABP no ERE deve ser realizada de forma modificada, com diminuição das horas de atividades síncronas, com melhor capacitação dos tutores e alunos quanto às TICs, 
melhor conhecimento das avaliações interativas online e das medidas profiláticas da fadiga zoom. O desafio maior é fazer com que os maiores aprendizados deste momento da pandemia da COVID-19 transformem, de forma duradoura, nosso processo educacional. Estudos adicionais, avaliando a implementação de novas estratégias para o melhor desempenho dos grupos tutoriais online neste centro universitário, devem ser realizados, bem investigadas as percepções dos discentes.

\section{Referências}

Al-Dous, K. K.; \& SAmaka, M. (2015). The design and delivery of hybrid PBL sessions in Moodle. International Journal of Education and Information Technologies (IJIET). 9:105-114. chrome-extension://oemmndcbldboiebfnladdacbdfmadadm/https://www.naun.org/main/NAUN/ educationinformat ion/2015/a282008-149.pdf

Bizarria, F. P. A; Tassigny, M. M., Bastos, A. T., \& Oliveira, A. G. (2013). Aprendizagem baseada em problemas em contextos de educação a distância. Revista EDaPECI. 13(2): 278-97. https://seer.ufs.br/index.php/edapeci/article/view/1289

Borges, M. C., Chachá, S. G. F., Quintana, S. M. Q., Freitas, L. C. C., \& Rodrigues, M. L. V. (2014). Aprendizado baseado em problemas. Medicina (Ribeirão Preto). 47(3): 301-7. https://www.revistas.usp.br/rmrp/article/view/86619

Dodge, M. (2020). 4 Signs You Have "Zoom Fatigue" (and What You Can Do About It). Jobillico. https://www.jobillico.com/blog/en/4-signs-you-have-zoomfatigue-and-what-you-can-do-about-it/.

Gibbs, T. (2020). The COVID-19 pandemic: provoking thought and encouraging change. Med Teach. 42 (7):738-40. https://www.tandfonline.com/doi/epub/10.1080/0142159X.2020.1775967?needAccess=true

Goldhame, M. E. J., Pusic, M. V., Co, J. P. T., \& Weinstein, D. F. (2020). Can Covid catalyze an educational transformation? Competency-based advancement in a crisis. $N$ Engl J Med. 383(11):1003-1005. https://www.nejm.org/doi/10.1056/NEJMp2018570

Hampel, R., \& Stickler, U. (2005). New skills for new classrooms: training tutors to teach languages online. Computer Assisted Language Learning. 18(4):311-26. https://www.tandfonline.com/doi/abs/10.1080/09588220500335455

Hodges, C.; Trust, T.; Moore, S.; Bond, A.; \& Lockee, B. (2020). Diferenças entre o aprendizado online e o ensino remoto de emergência. Revista da Escola, Professor, Educação e Tecnologia. 2:1-12. https://escribo.com/revista/index.php/escola/article/view/17

Innerdrive (2019). Are you experiencing zoom fatigue? Here's why. https://blog.innerdrive.co.uk/zoom-fatigue.

Kelley, K. W., Fowlin, J. M., Tawfik, A. A., \& Anderson, M. C. (2019). The role of using formative assessments in Problem-based Learning: a health sciences education perspective. Interdisciplinary Journal of Problem-Based Learning. 13(2):1-12. https://docs.lib.purdue.edu/ijpbl/vol13/iss2/6/

Leonard, T. (2020). The challenge of being resilient in the face of Zoom Fatigue. Statesman. https://www.statesman. com/opinion/20200427/ challenge-ofbeing-resilient-in-face-of-zoom-fatigue.

Mezzari, A. (2011). O uso da Aprendizagem Baseada em Problemas (ABP) como reforço ao ensino presencial utilizando o ambiente de aprendizagem Moodle. Rev Bras Educ Med. 35(1): 114-21. https://lume.ufrgs.br/handle/10183/81188

Moust, J. H. C., Berkel, H. J. M. V., \& Schmidt, H. G. (2005) Signs of Erosion: Reflections on Three Decades of Problem-based Learning at Maastricht University. High Educ. 50: 665-683. https://doi.org/10.1007/s10734-004-6371-z.

Nobre, C. F. C., \& Guerreiro, M. A. N. (2020) Ensinar e aprender em tempos da pandemia COVID-19. Saberes Plurais: Educação na Saúde 4 (2): 6-10. https://www.seer.ufrgs.br/saberesplurais/article/view/108896/59997

Office of Academic Affairs. (2020). How to mitigate and combat "Zoom Fatigue" in the online classroom. University of Nebraska Medical Center. https://connected. unmc.edu/blog/2020/05/05/how-to-mitigate-and-combat-zoom-fatigue/.

Pazin Filho, A.; \& Scarpelini, S. (2007). Estrutura de uma aula teórica I: Conteúdo. Medicina (Ribeirão Preto) 40 (1): 17-27. https://www.revistas.usp.br/rmrp/article/view/299

Petriglieri, G. (2020). How to deal with video calls, zoom fatigue, and remote relationships. Goop. https://goop.com/ wellness/mindfulness/zoom-fatigue/.

Ribeiro, L. R. C., \& Escrivão Filho, E. (2011). Avaliação formativa no ensino superior: um estudo de caso. Acta Scientiarum Human Social Sciences. 33(1):45-54. https://periodicos.uem.br/ojs/index.php/ActaSciHumanSocSci/article/view/9214

Rose, S. (2020). Medical student education in the time of COVID-19. JAMA. 323 (21):2131-32. https://jamanetwork.com/journals/jama/fullarticle/2764138

Sacasas, L. M. (2020). A theory of Zoom Fatigue. The Convivial Society. https://theconvivialsociety.substack.com/p/a-theory-of-zoom-fatigue.

Silva, A. C. O; Sousa, S. A., \& Menezes, J. B. F. (2020). O ensino remoto na percepção discente: desafios e benefícios. Dialogia 36: $298-315$. https://periodicos.uninove.br/dialogia/article/view/18383

Taylor, D.; Grant, J.; Hamdy, H.; Grant, L.; Marei, H.; \& Venkatramana, M. (2020). Transformation to learning from a distance. Dundee: MedEdPublish. 9(1):76. https://doi.org/10.15694/mep.2020.000076.1.

Tempski, P., Danila, A. H., Arantes-Costa, F. M., Siqueira, M. A. M., TorsanI, M. B., \& Martins, M. A. (2020). The COVID-19 pandemic: time for medical teachers and students to overcome grief. Clinics (Sao Paulo).75: e2206. https://doi.org/10.6061/clinics/2020/e2206. 
Research, Society and Development, v. 10, n. 5, e53510515280, 2021

(CC BY 4.0) | ISSN 2525-3409 | DOI: http://dx.doi.org/10.33448/rsd-v10i5.15280

Tolsgaard, M. G., Cleland, J., Wilkinson, T., \& Ellaway, R. H. (2020). How we make choices and sacrifices in medical education during the COVID-19 pandemic. Med Teach. 42(7):741-43. https://www.tandfonline.com/doi/full/10.1080/0142159X.2020.1767769

Torre, D. M., van der Vleuten, C., \& Dolmans, D. (2016). Theoretical perspectives and applications of group learning in PBL. Med Teach. 38 (2):189-95. https://www.tandfonline.com/doi/abs/10.3109/0142159X.2015.1009429 